\title{
Collaboration for Success in Crowdsourced Innovation Projects: Knowledge Creation, Team Diversity, and Tacit Coordination
}

\author{
Wenjun Zhou \\ University of Tennessee \\ wzhou4@utk.edu
}

\author{
Wangcheng Yan \\ University of Tennessee \\ wyan3@vols.utk.edu
}

\author{
Xi Zhang \\ Tianjin University \\ jackyzhang@tju.edu.cn
}

\begin{abstract}
When innovation projects are crowdsourced, individuals are allowed to form teams and collaborate to develop a successful solution. In this environment, teams will be competing with each other, as only the winning ones take the award home. Should a worker work alone, so that she or he does not need to share the award when she or he wins, or should she or he form a team for a better chance to win? In this paper, we studied the behaviors of workers in the context of crowdsourced innovation projects (CIPs). Building upon the theoretical framework of the organizational knowledge creation theory (OKCT), we linked team performance to team formation factors, including team diversity, team coordination, and task complexity. Our preliminary analysis showed that team coordination was an important factor for success. Team diversity in terms of connectivity was a positive factor towards better performance, whereas other factors were not significant. Our study indicates that workers in CIPs are likely to benefit from collaborations, connectivity diversity, and role diversity.
\end{abstract}

\section{Introduction}

Crowdsourcing refers to "using the Internet to outsource work to individuals" [15], which recently has been adopted as a new business model for innovation. Initially, crowdsourcing was introduced as a way to get small tasks done, such as image tagging (e.g., iStockphoto [3]), sentiment labeling (e.g., Amazon Turk [17]) and basic programming or editing tasks (e.g., taskcn [23]). These task-based crowdsourcing applications were supported by the economy of having work done cheaply [14] [18]. In these applications, the workers contribute small pieces of labor, which require minimal time and effort, and they did not have to collaborate with others to get the job done. More recently, crowdsourcing was also used for business innovation, such as new product design [2] [4] and problem solving [9] [24]. Idea contribution platforms, such as Dell's IdeaStorm [2], allow individuals to contribute ideas, and get rewarded if their ideas were adopted. Coming up with novel ideas does require users' basic knowledge of the company and the product, but the idea contribution action (i.e., posting comments on the forum) is still quite simple, without the need of collaborating with other contributors.

In this study, we study and test theories in knowledge creation and organization theories in the context of crowdsourced innovation projects (CIPs). CIPs are defined as projects that are crowdsourced to the public with the intention of innovation. Data and existing insights will be released to the public to develop a solution, such as algorithms, automated systems, or insights, to be used in the organization for the longer term. From the business organization's perspective, CIPs were crowdsourced for finding novel solutions more rapidly and more cheaply than hiring in-house experts [14]. It is also anticipated that novel analytical solutions will be proposed from the crowd, outside of the business organization. The most notable CIPs include the Netflix Prize, which has greatly improved the accuracy of predictions about whether a customer will enjoy a recommended movie, and inspired a new research domain in recommender systems. Since any person is allowed to try as long as she or he follows the basic rules, a cross-checking mechanism (i.e., comparing submission across different teams) was used for quality assurance. It takes tremendous amount of time to come up with a solution irrespective of quality. The workers have to download, analyze and create models to generate outputs for submission. Given the complexity of the innovation projects, workers in the crowd are allowed to form teams. Each team would then have one or more members. Forming teams and collaborate effectively would be a new research issue in the CIPs context.

In the new context of CIPs, we would like to test the applicability of knowledge creation theories and organization theories. The specific hypotheses we will test include: 1) whether team collaboration is a wise choice for workers in CIPs, and 2) whether more diverse teams are more likely to succeed. We will also explore whether externalization patterns could be 
found between teams (in spite of the competition). Understanding the workers' behaviors will provide the basis of theorizing the IS behaviors in this new context, and shed light on effective design of CIPs in the future.

Our hypotheses and implementations can be summarized as follows. First, we hypothesize that forming teams, instead of working alone is beneficial. Second, we believe forming more diverse teams is beneficial. The diversity considered in this study is the mix of new and old members. This can be extended in the future. Finally, we materialize the efficacy of tacit coordination by examining whether each pair of members maintain their collaboration in the future. Since there is a large variation in all competitions hosted, we consider the moderating effect of competition difficulty, inspired by the task complexity theory. Our empirical analysis has partially supported these hypotheses, and we also attempted to provide evidences to the four communications patterns in the organizational knowledge creation theory.

The novelty and contributions of this paper can be summarized as follows. Existing theories have not been tested in the new context of CIPs, which are based on an online platform that introduces both competition among teams (via the scoreboard) and knowledge sharing (via the discussion forum). Our study is the first to investigate the benefit of collaboration in CIPs, and the impact of other factors, such as team diversity and task complexity.

\section{Theoretical background}

In this study, we adopt knowledge creation and collaboration theories to build our research model, and test them in the context of CIPs. In particular, we adopt organizational knowledge creation theory (OKCT) as the overarching framework. According to the logic of tacit coordination theory and task complexity theory, we tested the relationship between performance and team diversity, task complexity, and team coordination.

\subsection{Organizational knowledge creation theory}

Organizational knowledge creation theory (OKCT) is a widely adopted theory in organizational and team knowledge management [29]. According OKCT, organizational knowledge can be classified as explicit knowledge (i.e., codified knowledge transmittable in formal, systematic language), and tacit knowledge (i.e., personalized knowledge that is hard to formalize or communicate, and rooted deeply in action, commitment and involvement in the context).

Teams that work on CIPs are formed ad hoc and informal, explicit knowledge may not be regularly codified within the team, but could be indirectly inferred by observing the team's performance. In the literature, the observable performance was commonly adopted as a proxy variable for explicit knowledge content [16][34]. Furthermore, tacit knowledge is personalized knowledge that is owned by individual members, not necessarily communicated formally (e.g., via meetings or documentation). She or he may acquire from or transfer to the team her or his tacit knowledge. Such within-team interactions, however, are hard to record or measure for CIPs.

In the context of CIPs, there are arguably two possible layers of organization: the team level (i.e., each team consists of one or more members) and the community level (i.e., all competing teams who are allowed to participate in a common discussion forum). The interactions among members of the same team normally happen offline, and may not be easily observable, as teams tend to form offline among people with existing social ties. In fact, previous study has shown that in terms of the social ties among participants of the CIPs, there is a large number of connected components in the social graph, that are completely disconnected from others [24]. Coupled with the competitive nature of the relation among the teams, we can reasonably believe that the participants tend to be isolated online between teams, and rather, more fully connected offline within teams. A typical scenario is that a group of graduate students, who are well acquainted with each other in class, decided to form a team and work together on a CIP together. As a result, even though we have no information at the lower level organization (i.e., within the team), we can possibly measure interactions at the community level (i.e., on the discussion forum across teams).

In the OKCT framework, organizational knowledge is created via four patterns of interactions: socialization, combination, internalization, and externalization [27]. Socialization is concerned with the exchange of tacit knowledge among individuals, and combination refers to the interactions to combine explicit knowledge as a group, through meeting and conversation or using information systems. Internalization refers to convert explicit knowledge into tacit knowledge, whereas externalization converts tacit knowledge into explicit knowledge.

At the team level, since most interactions happen offline, we have no access to the information. However, since forum posts are visible to the public and allows for knowledge sharing outside of the participating teams, some of the four patterns in the OKCT framework could be assessed at the community level. The discussion forums for CIPs are normally used for sharing knowledge or information. When users directly reply to each other's posts, we consider it an action of socialization. All users that participated in 
the forum discussions represent their teams who participated in combination (since all posts stay). Moreover, we can further separate forum posts into two categories: information acquiring and knowledge sharing. Information acquiring happens when a poster asks questions, seeking to internalize knowledge from the community. Knowledge sharing happens when a posts answers other people's questions, seeking to externalize the knowledge.

Note that interactions on the discussion forum are the primary interactions among the teams because they are not acquainted offline. We might contrast teams with an active forum member with those without. However, we cannot track the viewing log of participants, so it is hard to see which members combined knowledge from the forum without speaking up (i.e., posting a post). We can measure the socialization patterns in the CIPs community by the interactions on the forum.

\subsection{Team diversity}

The benefits of making teams in a competitive environment may seem obvious due to the general belief in better performance by more diverse teams, especially due to expertise coverage [16][21][34]. However, the theory has not been tested in CIPs. Possible benefits of forming teams with collaborators may include better chances to win (as empowered by the combined knowledge), and better retention (i.e., keep trying for a longer time), which in turn, will increase the chance to win, or at lease show improvement over time.

However, there are also drawbacks for forming a team. First, if they win, they have to share the money with fellow team members, the expected reward, therefore, is substantially reduced. Second, there is possible collaboration cost, such as communication overhead (e.g., scheduling a meeting), flexibility in realizing personal ideas, etc. This can be especially a concern when the team is huge. Last but not the least, the competition rules normally limit at most one submission per day. If four people work individually, they could submit four times in total. (The rule does forbid sharing data across teams, to avoid purposeful reverse engineering.)

H1: In CIPs, teams with multiple members perform better than teams with a single member.

We believe that forming teams rather than competing alone has a number of benefits due to the dynamics of knowledge creation and knowledge sharing process, however, this benefit relies on effective team forming and collaboration.

H2: In CIPs, for teams with more than one member, more diverse teams perform better.
Even though we know very little about the participants' background, and thus have no basis of judging for their skills diversity, we could measure other kinds of team diversity, i.e., experience, role, and connectivity diversities. In the context of small-group decisions, it was found that different diversity measures may have different effects on the decision efficacy [35]. In particular, in CIPs, we further established the diversity hypothesis into the following before testing them separately:

H2a (Experience diversity): teams with both older and newer members are more likely to perform better.

$\mathrm{H} 2 \mathrm{~b}$ (Role diversity): if some and not all of the team members use the forum, the team is more likely to perform better.

$\mathrm{H} 2 \mathrm{c}$ (Connectivity diversity): if the team has both more connected and less connected members (based on social network metrics such as connectivity), the team is more likely to perform better.

\subsection{Team coordination}

Coordination, either explicit or implicit, is the process of implementing teamwork, and is oftentimes defined as "the process of managing dependencies between activities" [26]. According to the visibility of coordination activities, Rico et al. [30] divide coordination into explicit coordination and tacit coordination. Explicit coordination refers to team members' intentional use of explicit manners, such as communication, to articulate plans, define responsibilities, negotiate deadlines, and seek information to accomplish the shared goals [7] [26]. In contrast, tacit coordination captures the capability of a team to act in concert by predicting the needs of the task and the team members, and adjust behavior accordingly, without overt communication [7] [30]. Therefore, the two basic dimensions of implicit coordination are anticipation and dynamic adjustment [30]. That is, team members can formulate other members' demands and actions by monitoring their progress with the task and their performance, and accordingly provide task-related information, proactively share tasks and adjust their own behaviors to keep in line with others' work without previous requests or direct notification.

The focus on explicit coordination is prevalent in previous research, which proves its undoubted importance. However, some studies acknowledge that explicit coordination only reveals one aspect of team coordination [30], and good coordination is nearly invisible [26] - but this does not mean that these two coordination mechanisms are opposite [30] [20]. Instead, prior research suggests that good coordination 
is a subtle mixture of explicit and tacit coordination [10], so these two kinds of coordination process can exist at the same time, and well-developed explicit coordination can leverage the development of tacit coordination.

Prior research about the explicit coordination mechanism suggests that team members coordinate explicitly by using task organization mechanisms or by communicating [8] [26]. However, task organization mechanisms such as planning and scheduling are less effective when the task has no or few routines because dependencies can no longer be managed in a programmed way [7]. Therefore, in our research about temporary CIPs, we focus on communication as the main tacit coordination mechanism, and it is also the dominant manner adopted by participants.

Tacit coordination is considered as the primary form of team coordination in CIPs because there is no formal organizational designation of roles or positions. However, we could possible probe into the roles according to other activity records (e.g., forum).

H3: Teams that coordinated better are more likely to perform better.

\subsection{Task complexity}

Task complexity has also been an important issue in IS theories, and applicable to our case. Task complexity is often considered as a moderator between teamwork and performance [1] [6] [12] [22] [28] [31]. In the context of CIPs, different competitions have vastly different levels of difficulty. Even though there is no direct measurement or indicator of level of difficulty, we can observe each competition's award amount (which is normally positively correlated with difficulty and participation), and the number of participating teams (which represents the intensity of competition.)

More specifically, task complexity can be included as a moderating variable. The level of difficulty is positively correlated with many of the following observable/unobservable factors: amount of price/reward expected; number of participating teams; whether the problem/data or the hosting company is interesting; and whether the problem is well defined or not. In this study, we simply use the number of participating teams (taken logarithm) as the proxy to task complexity. This is because it is not only easy to measure, but also directly affect the intensity of competition. After all, any team needs to rank on the top in order to receive the award. It also reflects the outcome of the other variables.

H4: The relative performance of a team is not dependent upon the difficulty of a competition.

\section{Research design}

Our study is based on a real-world dataset from a popular website that hosts CIPs. In this section, we will introduce the operationalization details.

\subsection{Data collection}

Kaggle.com is an online platform that provides crowdsourcing solutions for companies and non-profit organizations to get access to a large pool of analytical expertise. This platform hosts one of the world's biggest data science communities, which attracts experts from all over the world to participate in the CIPs, which are normally formulated as problemsolving competitions. Each competition is normally set up by providing a brief problem description, a page with links for data download and documentation, and a pre-specified set of performance metric, general participation rules, and awards information. The winning team needs to beat naive baselines or an existing model. This requirement ensures that the final result will show substantial improvement over triviality or the state of the art. The competition rules at Kaggle.com usually require that winners describe their winning model in exchange for a prize, which is typically a pre-specified amount of money, and may include other types of awards, such as conference travel, plaque, and job positions. The competition rules may also limit the maximum number of members in a team, and/or the maximum number of submissions each day. Kaggle.com has hosted more than 200 competitions in data science so far.

For each competition, there is also a public scoreboard and a discussion forum. The public scoreboard dynamically reflects teams' ranking, using a random subset of samples, when new submissions are being made to the website. Once a result (typically, the output from the team's model) is submitted to the website, teams will be ranked according to the performance on a random partition (e.g., 30\%) of the scoring instances and listed on the public scoreboard. The actual score on the full scoring set, which will be used to determine the winners at the end of the competition, is not released so that teams will not reverse engineer the results and over-fit the data. Given the competition among many teams, the best performing model typically improves the solution substantially. The discussion forum is where questions are asked and answered among all members of the Kaggle community, including Kaggle moderators. On the forum, there is a feature that allows users to click on the "thank" button to mark posts with useful 
information. The number of thanks to each post is visible to all forum participants.

The competition dataset was shared by Kaggle. This dataset includes the competitions information, the submission records, and the team-membership data. The competitions data include summary information of the competitions, such as award amount, number of teams participated, and number of entries submitted, which could be used to infer task complexity. The submissions data includes all teams' submission history and their ranking on the public scoreboard, saved in CSV files. Each submission is associated with a timestamp, the performance score (by the prespecified metric, such as misclassification rate or $\mathrm{F} 1$ measure), and the temporary (i.e., at the time of submission) and final (i.e., at the end of the competition period) ranking. After filtering out nonpublic competitions and those that were still in progress at the time of data collection, our preliminary dataset included 40,377 submission records to 57 competitions.

A second part of the dataset consists of the forum discussion activities, which was crawled using Python. We were able to clean and match 44 of the competitions to our preliminary set of competitions. The crawled information was then formatted into a CSV table, where each row corresponds to a post (including the initiating post and the follow-up replies), and the columns include the author (identified by username), the thread (identified by post title), the sequence number in the thread, and the number of "thanks" received from others. We can track each individual member's activities on the forum by their identifiable user names. Different from the public scoreboard data, the forum posts are submitted by individual team members, and thus we can differentiate the roles of those individuals in the team (i.e., whether a forum user, who communicates outside of the team with the whole community). Even though we have not performed a text mining and/or content analysis on the posts, we were able to easily quantify the value of content contributed by each user using the number of "thanks". In the future, we can also do text mining to find out the quality, content, or contribution of each post.

\subsection{Extraction of social network metrics}

A social network is typically modelled as a graph structure, where nodes represent actors of the system, and edges represent their relations. As a result, many graph based methods and tools are applicable for solving problems formulated in the social network context. A thorough text of related concepts and examples may be found in [13] or [5]. Extracting the wisdom of the crowd through social network analysis has also been found to be effective in many applications [10][32].

Since each team may have one or more members on Kaggle (and a member can only participate in one team that submits to a competition), the relationship between teams and members may be represented and analyzed as a bipartite graph. The bipartite graph, also known as two-mode network or affiliation network, is a special type of graph that is particularly suitable for modeling the relationship between two types of entities [25]. It is common practice to project a two-mode network into one mode, so that existing (one-mode) network analysis techniques may be readily applied. This approach is particularly useful when the primary interest is on the relationship among one of the modes. In our case, it is interesting to look into the social relation among individual members to assess their connectivity.

The team-membership data were used to create the affiliation network, based on which we constructed the social network among individual participants. Essentially, we link two individuals in the social graph if they have ever collaborated in any one or more competition. Our preliminary dataset included 30,142 links among 26,971 teams that involve 19,277 unique members. Using the igraph package in $\mathrm{R}$, we were able to easily create a bipartite graph between teams and members, map it into the member-member social network, and then calculate the degree centrality (i.e., number of directly connected members) for each member. Finally, we calculated the variance in team member's degree to represent the team's connectivity diversity.

\subsection{Partially observed team characteristics}

We would like to infer the efficacy of the team's knowledge creation process, which is reflected in the performance (e.g., ranking or score) or activities (e.g., number of attempts) of the teams. Even though there is no formal explicit and tacit knowledge data available, we started by using team performance as a proxy variable to represent the amount of knowledge.

Tacit or implicit coordination is not observable, so we began by quantifying the overall compatibility between pairs of team members. Even though there is no chance of surveying team member's satisfaction during the collaboration process, we could observe the team composition to see if the members continued to collaborate in the future. A team with good tacit coordination should result in positive collaboration experience, and the members are more likely to continue to collaborate in future. As a result, for each pair of members in a team, we calculated the ratio 
between the number of competitions they collaborated and the number of all completions that either of them participated (i.e., the Jaccard coefficient) afterwards.

Since tacit coordination is unobservable, we could build a hidden Markov model (HMM) to represent the "health" status of a team. HMM was successfully used in the literature to infer patients' health statuses based on their online activities [33]. Evolution of team performance over time can be tracked and used as external evidence of collaboration status. We adopted HMM to estimate the latent satisfaction for team performance.

\subsection{Operationalization of variables}

For each competition, we summarized a number of variables for each participating team. Table 1 provides definitions and operationalization of variables that we included in modeling.

Table 1: Operationalization of variables $(\boldsymbol{i}=$
team, $\boldsymbol{j}=$ competition)
\begin{tabular}{|l|l|l|}
\hline Variable & Description & Measurement \\
\hline Dependent Variable: Team Performance \\
\hline$P_{i j}$ & $\begin{array}{l}\text { Team } \\
\text { Performance }\end{array}$ & $\begin{array}{l}\text { Percentile (ranging from 0 to } 1, \\
\text { where } 1 \text { means the best } \\
\text { performance) of the best score } \\
\text { of team } i \text { in competition } j \\
\text { amongst all submissions on the } \\
\text { scoreboard. }\end{array}$ \\
\hline Independent Variable: Team Diversity \\
\hline$V e_{i}$ & $\begin{array}{l}\text { Experience } \\
\text { Diversity }\end{array}$ & $\begin{array}{l}\text { Entropy (0-1) of the proportion } \\
\text { of new members. }\end{array}$ \\
\hline$V r_{i}$ & $\begin{array}{l}\text { Role } \\
\text { Diversity }\end{array}$ & $\begin{array}{l}\text { Entropy (0-1) of the proportion } \\
\text { of forum users. }\end{array}$ \\
\hline$V c_{i}$ & $\begin{array}{l}\text { Connectivity } \\
\text { Diversity }\end{array}$ & $\begin{array}{l}\text { The variance in members' } \\
\text { degree centrality in the social } \\
\text { graph. }\end{array}$ \\
\hline Independent Variable: Team & Coordination \\
\hline$C_{i j}$ & $\begin{array}{l}\text { Team } \\
\text { Coordination }\end{array}$ & $\begin{array}{l}\text { The average of the pairwise } \\
\text { satisfaction score among team } \\
i \text { 's members at the end of } \\
\text { competition } j\end{array}$ \\
\hline Independent Variable: Task Complexity \\
\hline$D_{j}$ & $\begin{array}{l}\text { Competition } \\
\text { Difficulty }\end{array}$ & $\begin{array}{l}\text { Number of teams that } \\
\text { participated in competition } j, \\
\text { taken logarithm }\end{array}$ \\
\hline
\end{tabular}

The dependent variable, team performance, is measured as the percentile of the best score of team $i$ toward competition $j$, amongst all submissions to this competition. In order to build a regression model to explain team performance using other variables, we transformed it into the logit score, so that the dependent variable follows a Normal distribution.

The first independent variable, team diversity, could be measured in various ways, such as experience diversity, role diversity, and connectivity diversity. For experience diversity, we calculated the entropy based on the proportion of new members among all members of team i. A new member is defined as one who was never part of a team that made a submission to any other competition before the request date. Note that there is a large number of teams with just one member, for which the diversity is zero. Combined with other teams that have either new users only or experienced users only, the number of teams with some diversity is very small. We then decided to dichotomize this variable: whether the team has diversity or not. Similar treatment was done to role diversity as the entropy of forum users. The connectivity diversity is measured as the variance in degree centralities of team members when assessed in the context of their collaboration social network.

The second independent variable, team coordination, is calculated as the average of all pairwise coordination satisfaction scores. The coordination satisfaction score of any given pair of members, say, A and B, is calculated as the number of competitions that $\mathrm{A}$ and $\mathrm{B}$ completed together later than competition $\mathrm{j}$, divided by the total number of competitions that either A or B participated later than competition $\mathrm{j}$ (up to the end of the study period).

The third independent variable, task complexity is measured by the number of unique teams that participated in the competition, taken logarithm. The actual task complexity was not directly measurable. The most accurate indicator would be the award amount, as judged by the competition sponsor. However, such award amount is also a function of the sponsor's brand value and the importance of the problem to the sponsor. The number of participating teams, therefore, is the most direct and practical to measure how difficult it is to win. Since the raw values have a highly skewed distribution, we started by taking the log transformation. After looking at the distribution of the transformed values (i.e., log counts), we decided to discretize into a three-level ordinal variable. Namely, high, medium, and low levels of difficulty.

\subsection{Empirical Model}

Our empirical model can be summarized as follows, where we vaguely use $\mathrm{V}$ to represent one or more types of team diversity:

$$
\log \left(\frac{p_{i j}}{1-p_{i j}}\right)=\beta_{0}+\beta_{1} \cdot V_{i}+\beta_{2} \cdot C_{i j}+\beta_{3} \cdot D_{j}+\varepsilon_{i j} .
$$

Our goal is to explain teams' performances by the team's diversity, coordination, and the competition's level of difficulty. Note that according to our data processing setup, experience diversity is a dummy variable, and competition difficulty is a three-level categorical variable. 


\section{Findings}

In this section, we report initial findings. Overall, we found that collaborations are helpful. Teams that involve multiple members perform much better than those with just one member. Using one competition as an example, and by modeling the latent states of each team over time, we found that collaboration is particularly helpful for teams that rank in the middle. Our comprehensive empirical models indicate that team coordination and connectivity diversity are positively associated with performance, whereas the experience diversity is not. The competition difficulty was found insignificant.

\subsection{Collaboration is good for CIPs}

The first thing we looked at was the team size. In our dataset, the team size had a highly skewed distribution. More specifically, there were 10346 onemember teams, 512 two-member teams, 162 threemember teams, 51 four-member teams, 27 fivemember teams, and the remaining 32 teams with six or more members. Since the distribution is so skewed, we combined the teams with more than one member, and focused on comparing between single-member teams and multi-member teams.

We noticed was that it was rewarding to team up with others rather than working alone. When comparing teams with just one member (called "singles," the same hereafter) and those with multiple members (called "multiples," same hereafter), we found that the former $(\mathrm{n}=10346)$ performs much poorer than the later $(\mathrm{n}=784)$.

More specifically, the singles teams have an average of 0.013 in logit scores (that is, roughly $50.3 \%$ of other submissions were worse than them) of percentiles $(\mathrm{SD}=1.729)$; and the multiples group has an average of 0.744 in logit scores (that is, roughly $67.8 \%$ of other submissions were worse than them) of percentiles $(\mathrm{SD}=1.960)$, with a low $\mathrm{p}$-value. It is not surprising that a test with so large a sample size tends to be highly significant, however, the gap in the average performance (i.e., effect size) is quite large, too. This indicates that it is more likely to succeed when teaming up with others, rather than working alone. Therefore, $\mathrm{H} 1$ is well supported.

\subsection{Collaboration has different effects for teams in different latent states}

Thinking about the root why teaming up would help competitors perform better, we used the HMM model to estimate the latent state of each tem. Using one competition as an example, while tracking the progress over time, we categorize the "health" of each team into one of the following three levels: Good, Medium, and Bad. HMM is a commonly used model to estimate a latent variable that may change over time. It represents all the unobservable information within the team that could lead to externally reflected team performances (e.g., ranking, or logit score of percentiles).

Table 2 and Table 3 are the transition matrices among the three states, for singles teams and multiples teams, respectively. We can find several patterns by comparing the two transition matrices.

Table 2: Transition Matrix for Singles

\begin{tabular}{|l|c|c|c|}
\hline & Good & Medium & Bad \\
\hline Good & 0.80 & 0.20 & 0.00 \\
\hline Medium & 0.00 & 0.33 & 0.67 \\
\hline Bad & 0.22 & 0.00 & 0.78 \\
\hline
\end{tabular}

Table 3: Transition Matrix for Multiples

\begin{tabular}{|l|c|c|c|}
\hline & Good & Medium & Bad \\
\hline Good & 0.57 & 0.00 & 0.43 \\
\hline Medium & 0.26 & 0.74 & 0.00 \\
\hline Bad & 0.00 & 0.39 & 0.61 \\
\hline
\end{tabular}

First, for mediocre players, if they stay single, they have very little chance to get to the good level, and a high chance $(67 \%)$ to become bad. However, if they team up with others, their team status has quite some probability (26\%) to become good, and very low probability to become bad. Thereby, for medium level workers, it would be quite beneficial to form or join a team.

Moreover, for good workers, if they stay single, they have very low probability to become bad. However, if they form a team with others, their chances of staying good reduces from $80 \%$ to $57 \%$, and their chances to become bad also increases tremendously to a (43\%). It is possible that people who are proficient working alone (i.e., good singles) would more easily manage the work on their own and avoid the collaboration overhead.

Finally, workers in the bad state are likely to stay bad $(78 \%)$, or become good $(22 \%)$ when they work alone. However, if they were part of a team, they have very little chance to become good, in spite of a reduced chance $(61 \%)$ to stay bad. This indicates that "bad" teams do not benefit from collaboration.

\subsection{Positive factors for success}

After operationalizing the variables, we found a sample size of $n=334$ teams with complete data, upon 
which our regression analysis will be performed. The teams in our dataset consists of $39.5 \%$ high collaboration satisfaction, $28.4 \%$ high experience diversity, and $43.7 \%$ connectivity diversity cases. Moreover, the difficulty levels of competitions are $18.0 \%$ high, $45.2 \%$ medium, and all others low.

Table 4: Regression Analysis Results

\begin{tabular}{|l|r|r|r|r|}
\hline Variable & \multicolumn{1}{|c|}{ Est. } & \multicolumn{1}{c|}{ S.E. } & \multicolumn{1}{c|}{ t } & \multicolumn{1}{c|}{ p } \\
\hline C & 0.249 & 0.113 & 2.21 & $\mathbf{0 . 0 2 8}$ \\
\hline Ve & -0.128 & 0.124 & -1.03 & 0.305 \\
\hline Vc & 0.538 & 0.112 & 4.81 & $<\mathbf{0 . 0 0 1}$ \\
\hline D=High & 0.105 & 0.187 & 0.56 & 0.575 \\
\hline D=Low & -0.224 & 0.156 & -1.44 & 0.151 \\
\hline
\end{tabular}

The regression modeling results are reported in Table 4. We found that team coordination satisfaction $(\mathrm{p}=0.028)$ and connectivity diversity $(\mathrm{p}<0.001)$ were statistically significant, whereas all other variables were insignificant. The results support $\mathrm{H} 2 \mathrm{~b}$ and $\mathrm{H} 2 \mathrm{c}$ and does not support H2a. Note that competition difficulty was insignificant in all cases, meaning that no matter whether the competition is hard or easy, the same conclusions hold.

Table 5: Regression Analysis Results with Subsample (teams with forum users)

\begin{tabular}{|l|r|r|r|r|}
\hline Variable & Est. & \multicolumn{1}{|c|}{ S.E. } & \multicolumn{1}{c|}{ t } & \multicolumn{1}{c|}{ p } \\
\hline \multicolumn{5}{|c|}{ Model with Role Diversity } \\
\hline C & 0.096 & 0.153 & 0.63 & 0.532 \\
\hline Ve & -0.158 & 0.165 & -0.96 & 0.340 \\
\hline Vc & 0.534 & 0.155 & 3.44 & $<\mathbf{0 . 0 0 1}$ \\
\hline D=High & 0.004 & 0.264 & 0.02 & 0.987 \\
\hline D=Low & -0.415 & 0.206 & -2.01 & $\mathbf{0 . 0 4 6}$ \\
\hline Vr & 0.625 & 0.170 & 3.68 & $<\mathbf{0 . 0 0 1}$ \\
\hline \multicolumn{5}{|c|}{ Model without Role Diversity } \\
\hline C & 0.099 & 0.159 & 0.62 & 0.534 \\
\hline Ve & -0.169 & 0.171 & -0.99 & 0.324 \\
\hline Vc & 0.623 & 0.159 & 3.91 & $<\mathbf{0 . 0 0 1}$ \\
\hline D=High & -0.109 & 0.273 & -0.40 & 0.689 \\
\hline D=Low & -0.329 & 0.213 & -1.55 & 0.124 \\
\hline
\end{tabular}

With a subsample of 44 competitions, we attempted to test whether role diversity, in terms of who communicates to the community at the forum, was a significant factor. Table 5 reports the regression results with and without role diversity as an independent variable. Note that our sample size has been further reduced, but connectivity diversity remains highly significant. The role diversity is found significant, too, but team coordination, no matter whether role diversity is involved, is no longer significant within this subsample. This likely indicates that for this subsample (i.e., those with forum data) role diversity is a much more predictive independent variable than team coordination. This is reasonable because, by our operationalization, a team has role diversity if some (not none, and not all) members communicates externally via the forum. Such action indicates likely explicit coordination, which discounts the value of implicit coordination.

\subsection{Evidences in interaction patterns}

At the community level, we wanted to check the interaction patterns between team members and the community. In particular, did the performance correlate with contributions to outside of the team (i.e., in the forum)? Figure 1 shows a comparison in terms of number of the composition of better or worse performing teams (i.e., whether their performance was among the top half of participating team) by different levels of forum contribution. The forum contribution is measured as the average number of thanks per post. There is a large group of teams who either do not use the forum, or receive no thanks on average (first bar). We see that a majority of these teams belong to the underperformer group. The better performing groups take larger proportions for groups with more forum contributions. This is consistent with one would expect: those who create more knowledge internally are more likely to externalize to the community.

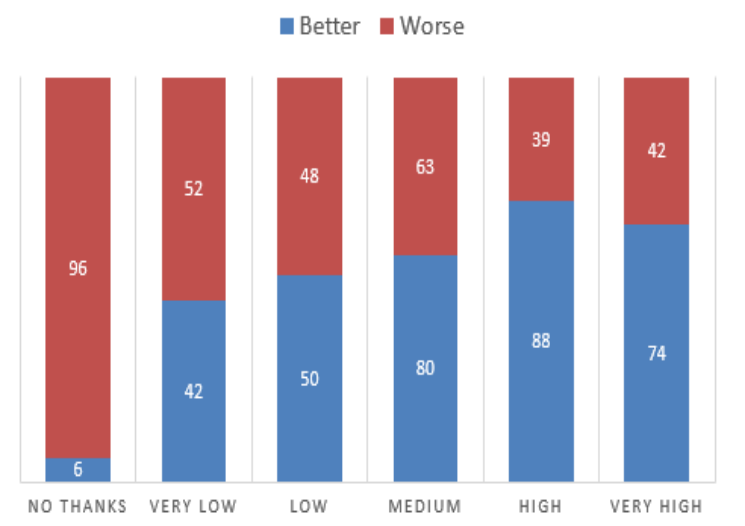

Figure 1: Performance vs. forum contribution

\section{Discussions and implications}

Our preliminary analysis indicated that in the context of CIPs, team coordination is likely a factor for success in this environment. Even though collaborating with others is not required, competing as a team is likely is better choice, especially for teams with medium level of performance. When they do, they are more likely to perform better, and stay active for longer time, which in turn, increases the chance of winning. 
Our analysis did not find support in the benefits of experience diversity. The same results hold irrespective of the level of difficulty. However, further exploration of the data, including other variable operationalization approaches, might be worthy of further investigation.

\subsection{Implications for research and practice}

Understanding the workers' behavior helps us better design an innovation project. In particular, our analysis supports the practice of allowing workers to form teams, or work alone if they feel more beneficial. Our findings in significant benefits in connectivity and role diversity may also indicate that setting different roles (more/less connected to others) or responsibilities (some use forum to communicate with the outside) would be beneficial.

New theories are needed in the new context of CIPs. There are inherent differences in different types of crowdsourcing tasks and thus the user commitment, responsibility in quality control and incentives are all different. Informed by this study, we should be able to further develop theoretical analysis on optional project design. The current literature all assume simple crowdsourced tasks and tasks like all-pay auctions.

\subsection{Challenges and future research}

Even though a team is like a team in the organization, and the team members can collectively decide which projects to spend time on, the crowdsourcing market might be quite different/novel in contrast to teams in an organization. Moreover, there is a two-layer community in a competition environment. This scenario is also possible in a company with many teams who work on related problems, and can compete for projects. The OKCT did not consider the two-layer framework and thus could be extended. We could not fully track the interactions within teams which can be a limitation. In the future, we are interested in developing new theories in the context of open innovation; and empirically and theoretically analyze the open innovation design for achieving organizational goals in innovation. Since members work on different projects with different people over time, it will also be interesting to study the knowledge spillover effect. For example, how do knowledge transfer from one team to another through a member's participation?

More thorough intra-team analysis will be insightful for identifying the different roles and teamlevel decision making processes. First, how do each team decide to work on which CIPs? When several competitions are open concurrently, a team's decision on taking part in one competition rather than another may help us understand their preferences [19]. We will infer the utility function of each project to a team, which is set up as a monotonous and quasi-concave function depending on reward types including the amount of money award, the number of award types, the level of kudos, the knowledge required by the project. Therefore, a team can be classified into a certain type according to preference characteristics. Inferring the utility function of each project to a team, according to their participation behaviors, will be useful for predicting the sensitivity of incentives. The outcome of will inform decision makers for developing new crowdsourcing projects in a cost effective way. For example, for a given analytical problem, how much do I have to offer in order to collectively improve my prediction by $10 \%$ ?

Finally, it will be interesting to extract individual metrics from forum posts, using text mining techniques to infer each individual's incentive, role and activeness in the team(s).

\section{References}

[1] Anand, G., P. T. Ward, and M. V. Tatikonda (2010). "Role of Explicit and Tacit Knowledge in Six Sigma Projects: An Empirical Examination of Differential Project Success," Journal of Operations Management 28:303-315

[2] Bayus, B. L. (2013). "Crowdsourcing New Product Ideas over Time: An Analysis of Dells IdeaStorm Community." Management Science 59(1):226-244.

[3] Brabham, D. C. (2008). "Moving the crowd at istockphoto: The composition of the crowd and motivations for participation in a crowdsourcing application." First Monday 13(6).

[4] Dahl, D. W., C. Fuchs, and M. Schreier (2015). "Why and When Consumers Prefer Products of User-Driven Firms: A Social Identification Account," Management Science 61(8):19781988.

[5] Easley, D. and J. Kleinberg (2010). Networks, Crowds, and Markets: Reasoning About a Highly Connected World. Available at: http://www.cs.cornell.edu/home/kleinber/networksbook/.

[6] Easton, G. S. and E. D. Rosenzweig (2015). "Team Leader Experience in Improvement Teams: A Social Networks Perspective,” Journal of Operations Management 37:13-30

[7] Espinosa, J. A. and E. Carmel (2004). "The Impact of Time Separation on Coordination in Global Software Teams: a Conceptual Foundation." Softw. Process Improve. Pract. 2003; 8: 249-266

[8] Faraj, S., and Sproull, L (2000). "Coordinating expertise in software development teams," Management Science (46:12), pp 1554-1568. 
[9] Franke, N., M. K. Poetz, and M. Schreier (2014) "Integrating Problem Solvers from Analogous Markets in New Product Ideation," Management Science 60(4): 1063-1081

[10] Godart, C., G. Halin, J. C. Bignon, C. Bouthier, O. Malcurat, and P. Molli (2001). "Implicit Or Explicit Coordination Of Virtual Teams In Building Design.” Key Centre of Design Computing and Cognition, University of Sydney, 2001, pp. 429-434

[11] Golub, B. and M. O. Jackson (2010). "Naïve Learning in Social Networks and the Wisdom of Crowds," Microeconomics 2(1):112-149.

[12] Hærem, T., B. T. Pentland, and K. D. Miller (2015). "Task Complexity: Extending a Core Concept," Academy of Management Review 40(3):446-460.

[13] Hanneman, R. A. and M. Riddle (2005). Introduction to Social Network Methods. University of California, Riverside, Riverside, CA. Published in digital form at http://faculty.ucr.edu/ hanneman/.

[14] Horton, J. J. and L. B. Chilton (2010). "The Labor Economics of Paid Crowdsourcing," in: Proceeding of the 11th ACM Conference on Electronic Commerce (EC '10), pp. 209-218.

[15] Howe, J. (2006). "The Rise of Crowdsourcing". Wired. Available at http://www.wired.com/2006/06/crowds/. Retrieved on 6/14/2016.

[16] Hsu, J. S.-C., S.-P. Shih, J. C. Chiang, and J. Y.-C. Liu (2012). "The Impact of Transactive Memory Systems on IS Development Teams' Coordination, Communication, and Performance," International Journal of Project Management 30(3):329-340

[17] Kittur, A., E. H. Chi, and B. Suh (2008). "Crowdsourcing User Studies with Mechanical Turk," in: Proceedings of the SIGCHI Conference on Human Factors in Computing Systems (CHI '08), pp. 453-456.

[18] Kleemann, F., G. G. Vo $\beta$, and K. Rieder (2008). "Un(der)paid Innovators: The Commercial Utilization of Consumer Work Through Crowdsourcing," Science, Technology \& Innovation Studies 4:5-26.

[19] Kokkodis, M. and P. G. Ipeirotis (2014). "The Utility of Skills in Online Labor Markets," in: Proceedings of the 35th International Conference on Information Systems (ICIS)

[20] Kotlarsky, J., P. C. van Fenema, and L.P. Willcocks (2008) "Developing a Knowledge-Based Perspective on Coordination: The Case of Global Software Projects", Information \& Management, 45(2), pp. 96-108.

[21] Lee, Y.-N., J. P. Walsh and J. Wang (2015). "Creativity in Scientific Teams: Unpacking Novelty and Impact," Research Policy 44(3): 684-697

[22] Liu, S. (2015) "Effects of Control on the Performance of Information Systems Projects: The Moderating Role of Complexity Risk," Journal of Operations Management 36:46-62
[23] Liu, T., J. Yang, L. A. Adamic, and Y. Chen (2014) "Crowdsourcing with All-Pay Auctions: A Field Experiment on Taskcn,” Management Science 60(8):2020-2037

[24] Lu, K., W. Zhou, and X. Wang (2014). "Social Network of the Competing Crowd," in: Proceedings of the 2014 International Conference on Behavioral, Economic, and Socio-Cultural Computing.

[25] Luo, C., H. Xiong, W. Zhou, Y. Guo, and G. Deng (2011). "Enhancing Investment Decision in P2P Lending: An Investor Composition Perspective," in: Proceedings of the 17th ACM SIGKDD International Conference on Knowledge Discovery and Data Mining, pp. 292-300.

[26] Malone, T. W., and K. Crowston (1994). "The interdisciplinary study of coordination", Computing Surveys, 26(1), pp. $87-119$.

[27] Nonaka, I. (1994). A dynamic theory of organizational knowledge creation. Organization Science, 5(1):14-37.

[28] Peng, D. X., G. R. Heim, and D. N. Mallick (2014). "Collaborative Product Development: The Effect of Project Complexity on the Use of Information Technology Tools and New Product Development Practices," Production and Operations Management Science 23(8):1421-1438

[29] Polanyi, M. (1962). "Tacit Knowing - Its bearing on some problems of philosophy" Reviews of Modern Physics 34(4):601-616.

[30] Rico, R., M. Sánchez-Manzanares, F. Gil, and C. Gibson (2008). "Team Implicit Coordination Processes: A Team Knowledge-Based Approach," Academy of Management Review, 33(1):163-184.

[31] Roberts, T. L., P. H. Cheney, P. D. Sweeney, and R. T. Hightower (2004). "The Effects of Information Technology Project Complexity on Group Interaction," Journal of Management Information Systems, 21(3):223-247

[32] Shang, S., P. Hui, S. R. Kulkarni, and P. W. Cu (2011). "Wisdom of the crowd: Incorporating social influence in recommendation models." Proceedings of the 2011 IEEE 17th International Conference on Parallel and Distributed Systems (ICPADS'11). 835-840.

[33] Yan, L., and Tan, Y. (2014). "Feeling Blue? Go Online: An Empirical Study of Social Support among Patients," Information Systems Research 25(4):690-709.

[34] X. Zhang, P. O. de Pablos, and Q. Xu (2014). "Culture Effects on the Knowledge Sharing in Multi-National Virtual Classes: A Mixed Method," Computers in Human Behavior, 31:491-498.

[35] Zhou, L., D. Zhang, and Y. Sung, (2013). The Effects of Group Factors on Deception Detection Performance. Small Group Research, 24(3), 272-297, June. 